\title{
Carbon-Nanotube-Polymer Nanofibers with High Thermal Conductivity
}

\author{
Vitaliy Datsyuk, ${ }^{*}$ Svitlana Trotsenko ${ }^{a}$ and Stephanie Reich ${ }^{a}$ \\ ${ }^{a}$ Freie Universität Berlin, FB Physik, Institut für Experimental Physik, Arnimallee 14, \\ 14195 Berlin, Germany
}

Highly thermally conductive carbon nanotube/polybenzimidazole polymer nanofiber composites were produced by core-shell electrospinning. The in-plane thermal conductivity increased by factor of 50 for $1.94 \% w t$. carbon nanotubes in the composite nanofibers. The high thermal conductivity results from the excellent nanotube alignment in the core of the polymer fiber and the use of liquid crystal polybenzimidazole as a matrix and shell polymer.

Polymer composites are useful for heat dissipation in electronic packaging and other applications. Compared to metal matrices polymers are light weight, which is key for mobile applications [1]. Other advantages of polymers include chemical resistance, lower electrical conductivity and mechanical flexibility. Carbon nanotubes (CNT) are outstanding thermal conductors with conductivities up to $3000 \mathrm{~W} / \mathrm{mK}$ [2]. Randomly oriented CNTs in polymer composites, however, generally results in poor heat dissipation with $\lambda=0.1-1 \mathrm{~W} / \mathrm{mK}$ for up to $50 \%$ wt. CNT loading [3]. The thermal conductivity increases by an order of magnitude upon alignment of the nanotubes, e.g., by external fields or through flow-induced orientation. Many alignment techniques, however, either require functionalization of the CNTs or are incompatible with industrial composite production [4].

In this paper, we report a mat of CNT-filled nanofibers with in-plane thermal conductivity of almost $20 \mathrm{~W} / \mathrm{mK}$ for less than $2 \%$ wt. nanotube content. The nanofibers are produced by electrospinning of polybenzimidazole (PBI) loaded with carbon nanotubes. An important advantage of our technique is the use of pristine, as-grown CNTs. The nanotubes are aligned

\footnotetext{
*Corresponding Author: Tel: +49 30 83852155; E-mail:datsyuk@zedat.fu-berlin.de (V. Datsyuk)
} 
in the fiber by core-shell spinning and the liquid-crystal character of PBI; the tubes form a network for the efficient heat transport away from a source.

The CNTs used in this study were multiwalled carbon nanotubes (MWCNT) purchased from Bayer MaterialsScience (Baytubes C150P, 96.40\% purity). TEM images showed MWCNT with diameters $10-16 \mathrm{~nm}$ and lengths up to $1.5 \mu \mathrm{m}$. PBI (PBI Performance Product Inc.) is a heterocyclic thermoplastic with excellent thermal, chemical, and mechanical properties at room and cryogenetic temperatures [5]. We prepared a dispersion of MWCNT (4 \%wt.) in DMAc in the presence of a small amount (up to $2 \%$ wt.) of PBI. Thanks to its nitrogen groups and high molecular weight PBI acts as a dispersing agent and stabilizes the tubes [6]. It also regulates the viscosity of the spinable dispersion. The viscosity of the solution for electrospinning the inner and outer part of a core-shell fiber has to differ by one order of magnitude [7]. The MWCNT dispersion was obtained by ultrasonication for 30 min (output power $100 \mathrm{~W}$ ) with a Sonopuls HD3100 (Bandelin) equipped with a cup horn operating at 10 kHz. The dispersions were homogeneous and stable, having a dark ink-like appearance. For the polymer shell of the nanofiber we used a solution of PBI (15-20 wt\%) in presence of LiCl (0.8-1.1 \%wt.) with viscosities 20-175 Ps.

Electrospinning was conducted from a coaxial injector with separate pipelines for the inner and outer solution. The flow rates were varied independently for each solution; with a ratio 1:5 - 1:10 for the inner and outer pipeline. The nanofibers were collected on sulfurized paper where they formed 150 - $250 \mu \mathrm{m}$ thick mats. Polymer shells formed continuous nanofibers at flow rates 0.4 - $0.8 \mathrm{ml} / \mathrm{h}$; the applied voltages were $20-26 \mathrm{kV}$. 


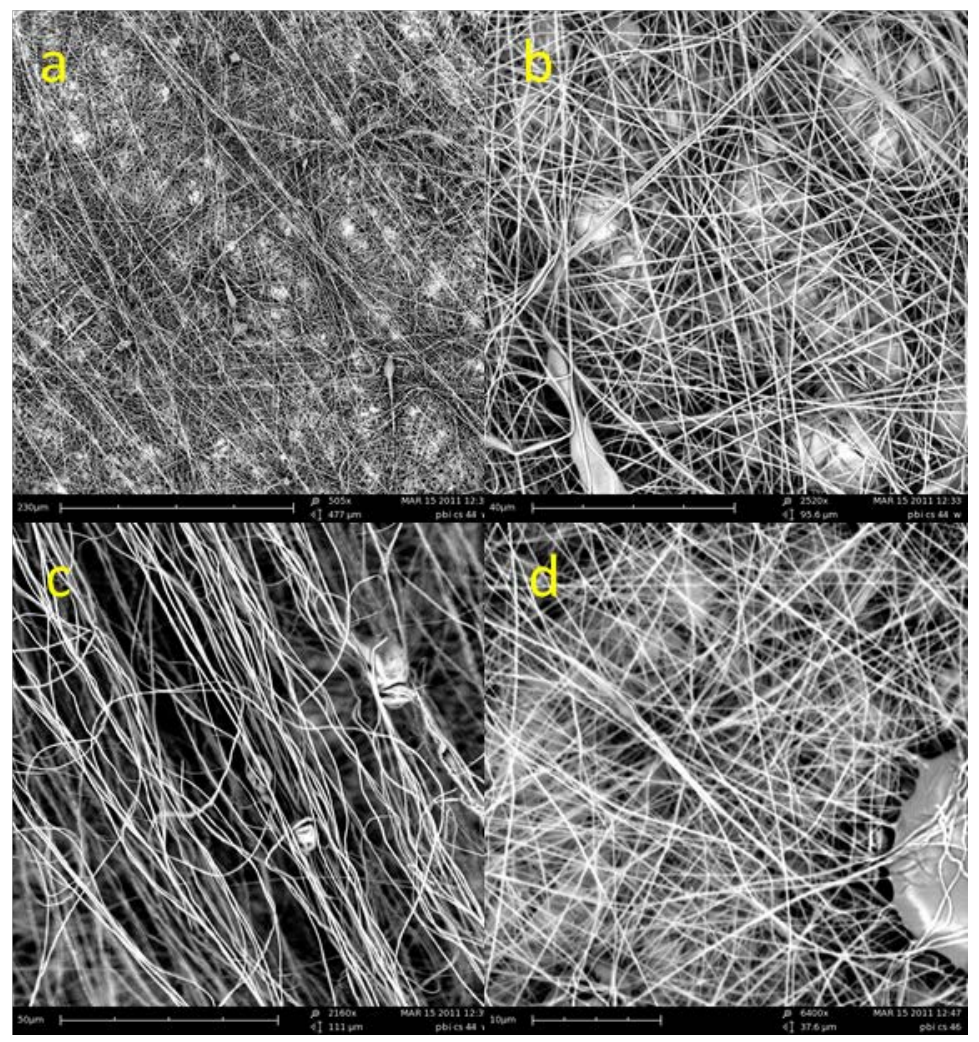

Figure 1. SEM images of the MWCNT-PBI nanofiber mats at $1.94 \% w t$. (a and b) and $4.44 \% w t$. ( c (on a drum) and d (on a flat)) MWCNT content.

The MWCNT content in the nanofibers composite ranged 1.9 - 4.4\%wt. Scanning electron microscopy images in Fig. 1 show that the nanofibers form a non-woven, highly porous material with a density $\sim 0.18 \mathrm{~g} / \mathrm{cm}^{3}$. The outer morphology of the nanofibers is independent of MWCNT concentration (Fig. 1b and d); in particular, the diameters remained constant as verified by TEM images, see Fig. 3 below. Typically, an increase in tube concentration results in increasing electrical conductivity and, hence, a smaller diameter in electrospinning. The concentration of our MWCNT fluid remained constant and the change in concentration was achieved by varying the inner and outer flow rates explaining the constant fiber diameters [8]. Electrospinning on a rotating drum collector lead to mats with oriented MWCNT-PBI fibers (Fig. 1c, 95\% alignment). 


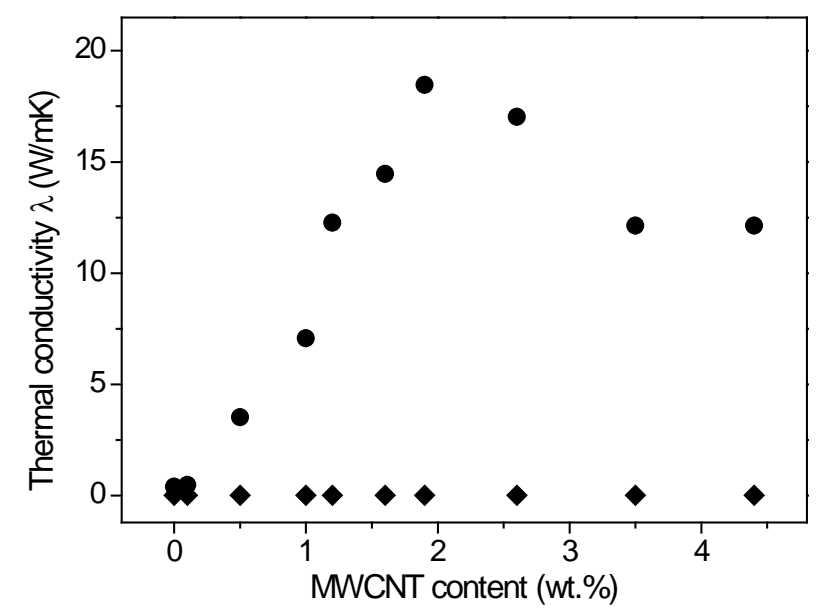

Figure 2. In-plane (circles) and through-plane (diamonds) thermal conductivities of the MWCNT-PBI fiber mats as a function of MWCNT content.

Samples of the MWCNT-PBI nanofiber mats were analysed with a HotDisk Transient Plane Source TPS 2500S thermal conductivity analyser (7577 sensor with $R=2.001 \mathrm{~mm}$ ). The inplane thermal conductivity of the CNT-PBI mats increased strongly from $0.5 \mathrm{~W} / \mathrm{mK}$ for $0.1 \%$ wt. to $18 \mathrm{~W} / \mathrm{mK}$ for $1.94 \%$ wt. CNTs embedded in the fibers, circles in Fig. 2. At higher loading the conductivity decreased and reached a plateau with $\lambda=12.0 \mathrm{~W} / \mathrm{mK}$. Perpendicular to the fibers the CNT-PBI mats were thermally insulating due to their high porosity; the through-plane conductivities were $0.010-0.014 \mathrm{~W} / \mathrm{mK}$ (Fig.2, diamonds).

The high thermal conductivity along the nanofibers in Fig. 2 is explained by CNT alignment at low concentration and a loss in alignment for higher loading. Two key ideas lead to the orientation of the MWCNTs in the polymer. First, electrospinning aligns the tubes through flow-confinement and charge-induced orientation. Second, the polybenzimidazole matrix aligns the nanotubes because of the lyotropic liquid crystalline properties of parapolybenzimidazole [9]. Liquid crystal polymers, additionally, increase the heat dissipation through the matrix as they are preferable for phonon travelling [10]. 


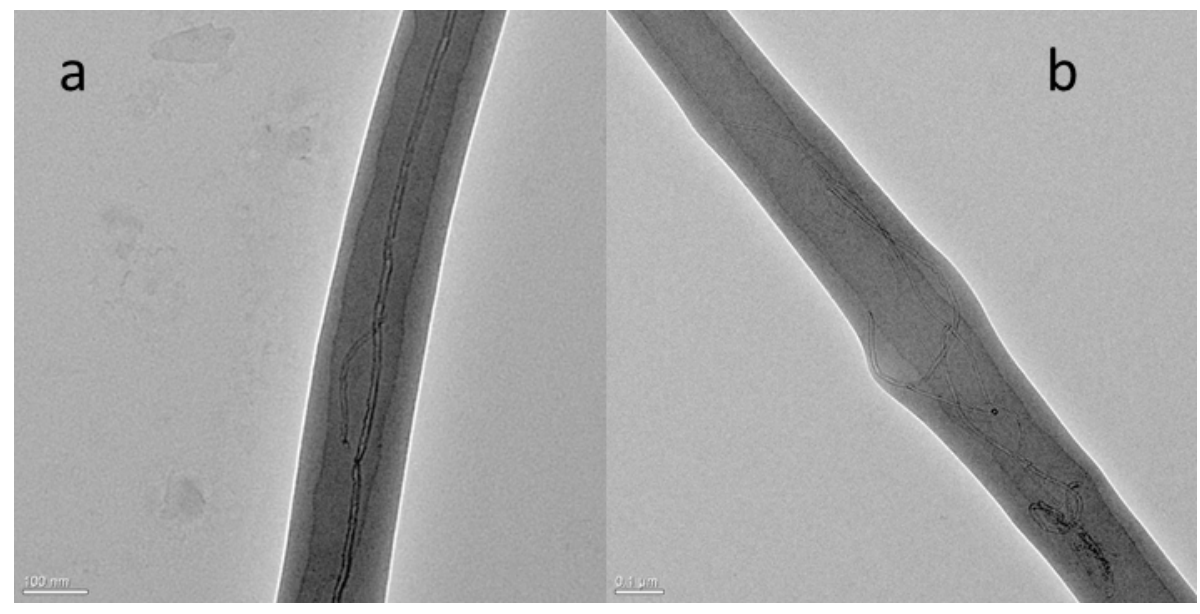

Figure 3. TEM images of the MWCNT-PBI core-shell nanofibers (a) $1.94 \%$ wt. MWCNT, the core shows well aligned nanotubes; (b) $4.40 \%$ wt. MWCNTs results in twisted and agglomerated tubes.

The lower in-plane thermal conductivity at higher CNT concentration in Fig. 2 points towards a decrease in alignment. Indeed, we observe an excellent network of well aligned MWCNTs in the sample with $1.94 \%$ wt. CNTs, see Fig. 3a. Increasing the loading to $4.4 \%$ wt. resulted in nanotube agglomerates that were twisted inside the fiber (Fig. 3b). Apparently, the large differences between the outer and inner flow rate necessary for small CNT concentrations help additionally through a flow-induced alignment [11]. Smaller gradients in the velocity and viscosity initiated an agglomeration visible in Fig. 3b. We attempted to further study tube orientation by polarized Raman scattering, but found the signal too weak. MWCNTs are poor scatterer; a detectable Raman intensity requires 10 \%wt. loading [12].

In summary, MWCNT-PBI nanofiber mats were synthesized by coaxial electrospinning. The CNTs showed a high degree of alignment at concentrations below 2 \%wt. The in-plane thermal conductivity of the composites reached $18 \mathrm{~W} / \mathrm{mK}$ at $1.94 \%$ wt nanotubes content, a factor of 50 increases compared to PBI. The fibers can be used as fillers in polymers composites and as coatings with a good conductivity in one or two directions and thermally insulating in the third direction.

We acknowledge funding by the Bundesministerium für Bildung und Forschung (grant number 03FO1022 and 16V0156). 


\section{References:}

[1] Byrne MT, Gun’ko YK, Recent Advances in Research on Carbon Nanotube-Polymer Composites, Adv. Mater. 2010; 22(15):1672-88.

[2] Hone J, Whitney M, Piskoti C, Zettl A, Thermal Conductivity of Single Walled Carbon Nanotubes Phys. Rev. B, 1999; 59(4):R2514-6

[3] Datsyuk V, Lisunova M, Kasimir M, Trotsenko S, Gharagozloo-Hubmann K et.al, Thermal transport of oil and polymer composites filled with carbon nanotubes Appl. Phys. A. 2011; 105(4):781-90;

[4] Xie XL, Mai YW, Zhou XP, Dispersion and alignment of carbon nanotubes in polymer matrix: A review, Mater. Sci. Engin. R, 2005; 49:89-112;

[5] Zhang L, Ni QQ, Shiga A, Natsuki T, Fu Y, Preparation of Polybenzimidazole/ functionalized carbon nanotube nanocomposite films for use as protective coatings, Polym. Engin. Sci., 2011; 51(8):1525-32;

[6] Okamoto M, Fujigaya T, Nakashima N, Individual Dissolution of Single-Walled Carbon Nanotubes by Using Polybenzimidazole, and Highly Effective Reinforcement of Their Composite Films, Adv. Funct. Mater. 2008; 18(12):1776-1782.

[7] Dror Y, Salalha W, Avrahami R, Zussman E, Yarin A L, et al. One-Step Production of Polymeric Microtubes by Co-electrospinning, Small, 2007; 3(6):1064-73.

[8] Salalha W, Dror Y, Khalfin RL, Cohen Y, Yarin AL, Zussman E, Single-walled carbon nanotubes embedded in oriented polymeric nanofibers by electrospinning. Langmuir 2004; 20(22):9852-5.

[9] Chung TS, A critical review of polybenzimidazoles: Historical development and futu-re R\&D. J. Macromol. Sci., Rev. Macromol. Chem. Phys. C, 1997; 37(2):277-301

[10] Picot JJC, Fredrickson AJ, Interfacial and electrical effects on thermal conductivity of nematic liquid crystals, Ind. Eng. Chem. Fundamen., 1968; 7(1):84-89 
[11] Wang M, Yu JH, Kaplan DL, Rutledge GC, Production of sub-micron diameter silk fibers under benign processing conditions by two-fluid electrospinning, Macromol. 2006; 39(3):1102-7.

[12] Lisunova M, Hildmann A, Hatting B, Datsyuk V, Reich S Nanofibres of CA/PAN with high amount of carbon nanotubes by core-shell electrospinning. Compos. Sci. Technol. 2010; 70(11):1584-8. 